\title{
Impact of MGMT Promoter Methylation as a Prognostic Marker in Patients with High Grade Glioma: A Single-Center Observational Study
}

\author{
Vinu Sarathy*, Srinivasa Belagutty Jayappa, Bhanu Lalkota, Kiran Pura Krishnamurthy, \\ Vishal Kulkarni, Samuel Luke Koramati, Nasiruddin Mohammad, Radheshyam Naik
}

Department of Medical Oncology, Health Care Global Enterprises Ltd., Bangalore, India

Email: *sarathy.vinu.88@gmail.com

How to cite this paper: Sarathy, V., Jayappa, S.B., Lalkota, B., Krishnamurthy, K.P., Kulkarni, V., Koramati, S.L., Mohammad, N. and Naik, R. (2019) Impact of MGMT Promoter Methylation as a Prognostic Marker in Patients with High Grade Glioma: A Single-Center Observational Study. Journal of Cancer Therapy, 10, 806-814. https://doi.org/10.4236/jct.2019.1010068

Received: May 31, 2019

Accepted: October 5, 2019

Published: October 8, 2019

Copyright $\odot 2019$ by author(s) and Scientific Research Publishing Inc. This work is licensed under the Creative Commons Attribution International License (CC BY 4.0).

http://creativecommons.org/licenses/by/4.0/

\begin{abstract}
Objectives: 1) To correlate the methylation status of the O-6-methylguanineDNA-methyltransferase (MGMT promoter gene) and response to alkylating agent-based treatment in high-grade gliomas. Background: The MGMT gene is epigenetically silenced by promoter hypermethylation in gliomas and this modification has emerged as a relevant predictor of therapeutic response. Methods: 20 cases of high-grade glioma were analyzed for MGMT promoter methylation by methylation-specific PCR. Response to treatment and overall survival data were recorded and data analysed. Results: MGMT promoter methylation was found in $60 \%$ of gliomas by methylation-specific PCR. The mean survival time of glioblastoma patients submitted to adjuvant therapy was significantly higher among patients with MGMT promoter methylation $(\mathrm{P}=0.035)$ and methylation status was an independent predictive factor that was associated with improved prognosis. Discussion and Conclusion: MGMT promoter methylation status was a more reliable predictor of response to adjuvant therapy and prognosis of high-grade gliomas. A subset of patients received irinotecan and bevacizumab in the second line setting and patients with unmethylated MGMT seemed to do better than the MGMT promoter methylated group.
\end{abstract}

\section{Keywords}

Glioblastoma, MGMT Promoter Methylation, MGMT Gene, Aklylating Agents, Temozolomide, Prognosis

\section{Introduction}

Patients with MGMT (O-6-methylguanine-DNA methyltransferase) promoter 
methylation have been associated with longer survival in high-grade glioma patients who have received treatment with alkylating chemotherapy in addition to radiotherapy.

This epigenetic silencing of the MGMT promoter region confers a survival advantage to patients who received carmustine or temozolomide along with radiation and as part of adjuvant therapy [1] [2].

The MGMT gene is located on chromosome 10 q26 and codes for a DNA repair protein which removes alkyl groups from the O6 position of guanine-a hotspot of DNA alkylation. If left unrepaired the DNA damage by chemotherapy especially at the O-6-methylguanine position triggers cytotoxicity and apoptosis [3] [4].

As a result, epigenetic silencing of MGMT promoter region is associated with loss of MGMT expression and reduced DNA repair activity [5] [6] [7].

GBMs (glioblastoma multiforme) are classified as grade $4 / 4$ by the WHO and have a very dismal prognosis in most patients surviving only 1 - 2 years despite aggressive management [8].

Although age, the extent of resection and performance status remain the most reliable prognostic markers in patients survival, MGMT expression has gained interest as a predictive marker for response to chemotherapy, especially with alkylating agents like temozolomide and carmustine.

The current standard treatment of high-grade glioma is based on a Phase III trial conducted by the EORTC, which showed a significant improvement in survival in the radiation plus concurrent and adjuvant temozolomide arm when compared to radiation alone [9].

From the subset analysis, the authors concluded that MGMT promoter methylation conferred a survival advantage in patients receiving temozolomide which was not observed in the only radiotherapy arm.

Interestingly, the time to progression of patients in the control arm (radiotherapy alone) also appears to be more favourable in the patient whose tumors had MGMT promoter methylation suggesting that this biomarker is associated with improved radiation response. This is more so relevant considering radiation response has been shown as a strong predictor of survival in patients with GBM [10].

Therefore, the aim of our study was to look for any correlation between MGMT methylation status and treatment outcomes in patients with glioma.

\section{Inclusion criteria}

Patients above the age of 18 years with tissue confirmed that the diagnosis of glioma was selected from our institution. All patients with tissue sufficient for MGMT promoter methylation assessment were considered evaluable. All samples were from patients with newly diagnosed glioma who had not received prior treatment.

\section{Exclusion criteria}

Grade I tumors and patients with a PS of 4 were excluded from our study. 


\section{Materials and Methods}

A retrospective analysis of the patient charts was done to collect patient data such as demographics, the extent of surgical resection, treatment modalities \& time to progression. Patients were treated with concurrent/adjuvant temozolomide post-surgery as part of standard therapy. Patients who had an unsatisfactory response or early disease progression post completion of primary treatment, received treatment with second line treatment which included bevacizumab and irinotecan.

Twenty cases with sufficient tissue for molecular analysis were identified. All patients were treated initially with temozolomide and concurrent RT. Patients whose disease response was unsatisfactory to first line chemo-RT or who progressed early received second line treatment in the form of irinotecan $\&$ bevacizumab.

\subsection{DNA Extraction/Bisulfite Treatment}

Routinely processed formalin-fixed, paraffin-embedded GBM samples were selected from the 20 cases. The hematoxylin and eosin-stained slides were reviewed by a neuropathologist, and appropriate blocks were selected for tumor. Following deparaffinization, DNA extraction was performed following which Bisulfite treatment was then performed on the methylation-specific qRT-PCR and determination of MGMT Promoter Methylation. qRT-PCR was performed using the eluted bisulfite-treated DNA. PCR reactions were set at $20 \mathrm{~mL}$ volumes using up to $5 \mathrm{~mL}$ of bisulfite-treated DNA, methylation-specific primers and probes.

The above results were then correlated with the patient variables, response to treatment and survival.

\subsection{Statistical Methods}

Descriptive and inferential statistical analysis has been carried out in the present study. Results on continuous measurements are presented on Mean SD (MinMax) and results on categorical measurements are presented in Number (\%). Significance is assessed at $5 \%$ level of significance. The following assumptions on data are made: 1) Dependent variables should be normally distributed; 2) Samples drawn from the population should be random, cases of the samples should be independent.

Chi-square/Fisher Exact test has been used to find the significance of study parameters on categorical scale between two or more groups, Non-parametric setting for Qualitative data analysis. Fisher Exact test used when cell samples are very small.

Overall survival curves were estimated by the Kaplan-Meier technique and compared with use of the two-sided log-rank test.

\section{Significant figures}

${ }^{+}$Suggestive significance (P-value: $0.05<\mathrm{P}<0.10$ ); 
${ }^{*}$ Moderately significant (P-value: $\left.0.01<\mathrm{P} \leq 0.05\right)$;

**Strongly significant ( $\mathrm{P}$-value: $\mathrm{P} \leq 0.01)$.

\subsection{Statistical Software}

The Statistical software namely SPSS 18.0, and R environment ver.3.2.2 were used for the analysis of the data and Microsoft Word and Excel have been used to generate graphs, tables etc.

\section{Results}

Baseline characteristics of the patients are represented in Tables 1-3. Most of our patients were above the age of 50 years, male and had a good performance status of 1 .

Out of the 20 patients studied, 12 patients had MGMT promoter methylation while 8 had an unmethylated status as depicted in Table 4.

As noted in Table 5, 75\% of the patients were diagnosed as glioblastoma multiforme

Table 1. Age in relation to MGMT status of patients studied.

\begin{tabular}{cccc}
\hline \multirow{2}{*}{ Age in years } & \multicolumn{2}{c}{ MGMT Status } & \multirow{2}{*}{ Total } \\
\cline { 2 - 3 } & Methylated & Unmethylated & $7(35 \%)$ \\
\hline$<50$ & $4(33.3 \%)$ & $3(37.5 \%)$ & $7(35 \%)$ \\
$50-60$ & $6(50 \%)$ & $1(12.5 \%)$ & $3(15 \%)$ \\
$71-70$ & $1(8.3 \%)$ & $2(25 \%)$ & $3(15 \%)$ \\
$71-80$ & $1(8.3 \%)$ & $2(25 \%)$ & $20(100 \%)$ \\
Total & $12(100 \%)$ & $8(100 \%)$ & $50.65 \pm 17.62$
\end{tabular}

Table 2. Gender in relation to MGMT status of patients studied.

\begin{tabular}{cccc}
\hline \multirow{2}{*}{ Gender } & \multicolumn{2}{c}{ MGMT Status } & \multirow{2}{*}{ Total } \\
\cline { 2 - 3 } & Methylated & Unmethylated & $7(35 \%)$ \\
\hline Female & $5(41.7 \%)$ & $2(25 \%)$ & $13(65 \%)$ \\
Male & $7(58.3 \%)$ & $6(75 \%)$ & $20(100 \%)$ \\
Total & $12(100 \%)$ & $8(100 \%)$ & \\
\hline
\end{tabular}

Table 3. Performance status in relation to MGMT status of patients studied.

\begin{tabular}{cccc}
\hline \multirow{2}{*}{ Performance status } & \multicolumn{2}{c}{ MGMT Status } & \multirow{2}{*}{ Total } \\
\cline { 2 - 3 } & Methylated & Unmethylated & \\
\hline 1 & $9(75 \%)$ & $3(37.5 \%)$ & $12(60 \%)$ \\
2 & $2(16.7 \%)$ & $5(62.5 \%)$ & $7(35 \%)$ \\
3 & $1(8.3 \%)$ & $0(0 \%)$ & $1(5 \%)$ \\
Total & $12(100 \%)$ & $8(100 \%)$ & $20(100 \%)$ \\
\hline
\end{tabular}


Table 4. MGMT status.

\begin{tabular}{ccc}
\hline MGMT Status & No. of patients & $\%$ \\
\hline Methylated & 12 & 60.0 \\
Unmethylated & 8 & 40.0 \\
Total & 20 & 100.0 \\
\hline
\end{tabular}

Table 5. MGMT status and grade of the tumor.

\begin{tabular}{cccc}
\hline \multirow{2}{*}{ Diagnosis } & \multicolumn{2}{c}{ MGMT Status } & \multirow{2}{*}{ Total } \\
\cline { 2 - 3 } & Methylated & Unmethylated & \\
\hline Glioblastoma Multiforme (GBM)-grade IV & $9(75 \%)$ & $6(75 \%)$ & $15(75 \%)$ \\
Anaplastic astrocytoma-grade III & $2(17 \%)$ & $2(25 \%)$ & $4(20 \%)$ \\
Gemistocytic astrocytoma-grade II & $1(8 \%)$ & $0(0 \%)$ & $1(5 \%)$ \\
Total & $12(100 \%)$ & $8(100 \%)$ & $20(100 \%)$ \\
\hline
\end{tabular}

$\mathrm{P}=0.437$, Not significant, Fisher Exact test.

and the remainder made up the anaplastic astrocytoma and gemistocytic astrocytoma groups.

As noted there was no correlation of MGMT promoter methylation status and grade of the tumour.

7 patients had a complete resection, 12 had subtotal resection and in one patient only a biopsy was possible as depicted in Table 6 .

They were more or less almost equally distributed between the MGMT methylated and non-methylated groups.

The patients with MGMT promoter methylated status had a higher percentage of response to treatment as compared to the MGMT unmethylated group. $50 \%$ of patients in the unmethylated group had progressive disease compared to only $17 \%$ in the methylated group. The following findings are represented in Table 7.

The following data shows a trend towards better responses in the MGMT methylated arm, however, $\mathrm{P}$-value is not significant.

11 out of the 20 patients had a relapse on follow up and received second line treatment.

18 out of the 20 patients could achieve some objective response following one and/or two lines of therapy. 2 patients were too frail to withstand therapy and were supported with palliative treatment.

Out of the 7 patients who had completed concurrent RT with Temozolomide and adjuvant temozolomide for 1 year, 5 of the patients had MGMT methylated status. Among the patients with progressive disease and treated with second line treatment irinotecan + bevacizumab, interestingly, patients with MGMT unmethylated status had a better response to irinotecan (5 patients) when compared to MGMT methylated status (1 patient). However, the above data do not meet statistical significance. The other forms of second line treatment administered 
are bevacizumab alone (2) and stereotactic radio surgery (1). Two patients were lost to follow up. The entire data is depicted in Table 8.

As evident from Table 9, more patients on the methylated MGMT status arm Table 6. Surgery in relation to MGMT methylation status.

\begin{tabular}{cccc}
\hline \multirow{2}{*}{ Surgery } & \multicolumn{2}{c}{ MGMT Status } & \multirow{2}{*}{ Total } \\
\cline { 2 - 3 } & Methylated & Unmethylated & \\
\hline Biospy & $1(8.3 \%)$ & $0(0 \%)$ & $1(5 \%)$ \\
Subtotal resection & $7(58.3 \%)$ & $5(62.5 \%)$ & $12(60 \%)$ \\
Total resection & $4(33.3 \%)$ & $3(37.5 \%)$ & $7(35 \%)$ \\
Total & $12(100 \%)$ & $8(100 \%)$ & $20(100 \%)$ \\
\hline
\end{tabular}

Table 7. Post-treatment MRI response to first line therapy and MGMT methylation status.

\begin{tabular}{cccc}
\hline \multirow{2}{*}{$\begin{array}{c}\text { Post RX Magnetic } \\
\text { resonance imaging }\end{array}$} & \multicolumn{2}{c}{ MGMT Status } & \multirow{2}{*}{ Total } \\
\cline { 2 - 3 } PR & $2(16.7 \%)$ & Unmethylated & \\
SD & $8(66.7 \%)$ & $4(0 \%)$ & $2(10 \%)$ \\
PD & $2(16.7 \%)$ & $4(50 \%)$ & $12(60 \%)$ \\
Total & $12(100 \%)$ & $8(100 \%)$ & $6(30 \%)$ \\
\hline
\end{tabular}

$\mathrm{P}=0.330$, Not significant, Fisher Exact test; $\mathrm{PR}$ - partial response, $\mathrm{SD}$-stable disease, $\mathrm{PD}$ - progressive disease.

Table 8. Objective treatment responses $\left(1^{\text {st }}\right.$ line $\left.+2^{\text {nd }} l i n e\right)$ and relation to MGMT methylation status.

\begin{tabular}{cccc}
\hline & \multicolumn{2}{c}{ MGMT Status } & Total \\
\cline { 2 - 3 } & Methylated (n= 12) & Unmethylated (n=8) & $(\mathbf{n}=\mathbf{2 0})$ \\
\hline Nil response & $1(8.3 \%)$ & $1(12.5 \%)$ & $2(10 \%)$ \\
Yes & $11(91.7 \%)$ & $7(87.5 \%)$ & $18(90 \%)$ \\
TMZ with RT $\rightarrow$ adj TMZ * 1 year & $5(41.7 \%)$ & $2(25 \%)$ & $7(35 \%)$ \\
Irinotecan+ bevacizumab & $1(8.3 \%)$ & $5(62.5 \%)$ & $6(30 \%)$ \\
Bevacizumab alone & $2(16.7 \%)$ & $0(0 \%)$ & $2(10 \%)$ \\
Lost follow up & $2(16.7 \%)$ & $0(0 \%)$ & $2(10 \%)$ \\
SRS & $1(8.3 \%)$ & $0(0 \%)$ & $1(5 \%)$ \\
\hline
\end{tabular}

TMZ—-temozolomide, SRS—sterotactic radiosurgery.

Table 9. Follow up and relation to MGMT promoter methylation status of patients studied.

\begin{tabular}{cccc}
\hline \multirow{2}{*}{ Follow up } & \multicolumn{2}{c}{ MGMT Status } & \multirow{2}{*}{ Total } \\
\cline { 2 - 3 } & Methylated & Unmethylated & \\
\hline Expired & $5(42 \%)$ & $6(75 \%)$ & 11 \\
On follow up till Dec. 18 & $7(58 \%)$ & $2(25 \%)$ & 9 \\
Total & $12(100 \%)$ & $8(100 \%)$ & 20 \\
\hline
\end{tabular}

$\mathrm{P}=0.035^{*}$, significant, Fisher Exact test. 
were still alive and doing well on follow up (58\%) as compared to patients with an unmethylated MGMT promoter phenotype (25\%). The P-value of 0.035 is significant.

The above data can be further illustrated and seconded by the Kalpan-meier estimates of overall survival (Figure 1) which show a better probability of survival in the MGMT promoter methylation group as compared to the unmethylated arm.

\section{Discussion}

We found that MGMT promoter methylation is associated with a favorable outcome after temozolomide chemotherapy in patients with newly diagnosed glioma. Our data suggest that the methylation status of the MGMT promoter may be a relevant predictor of benefit from temozolomide chemotherapy and may even have prognostic value.

Determination of MGMT promoter methylation status by methylation-specific PCR may allow for the selection of patients who are most likely to benefit from temozolomide treatment. Patients whose tumors are unmethylated at the MGMT promoter region appear to derive little benefit from the addition of temozolomide to radiotherapy. For these patients, alternative treatments with a different mechanism of action or methods of inhibiting MGMT should be developed [11] [12].

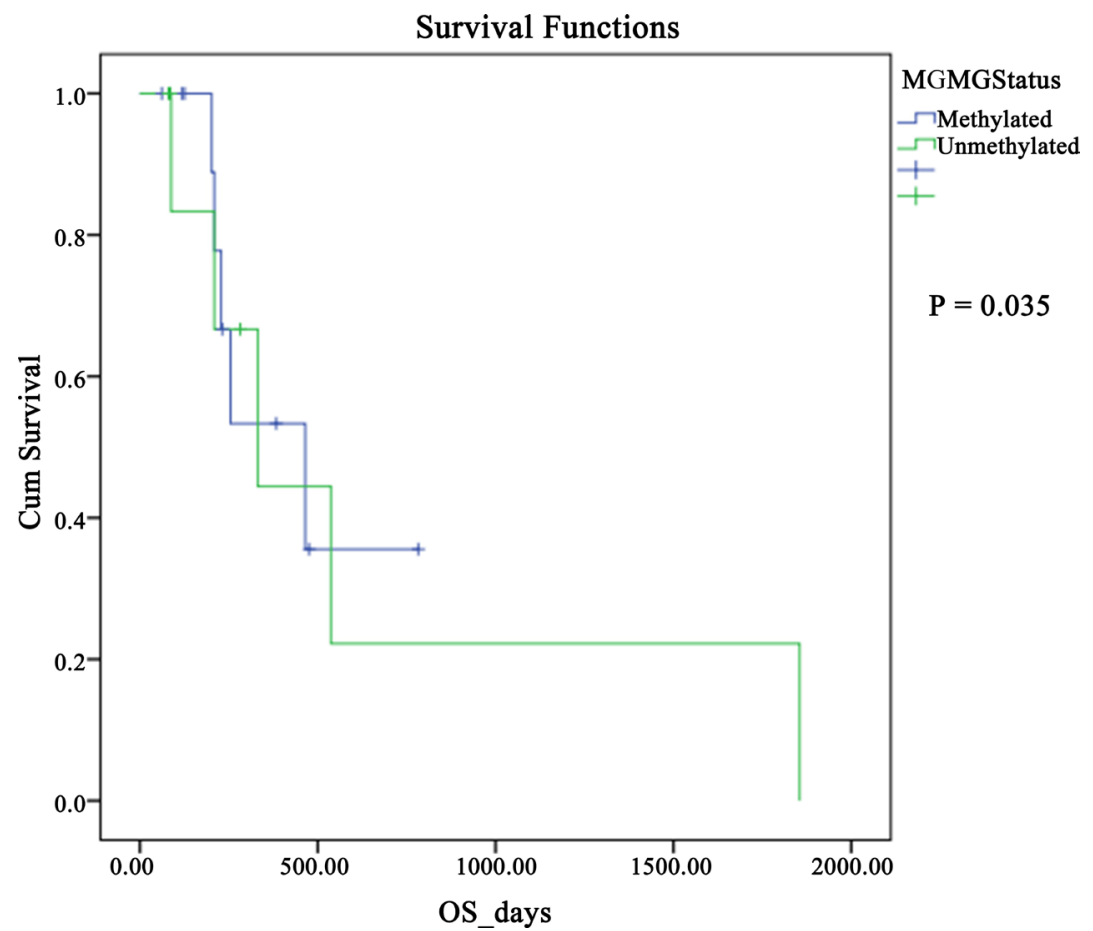

The difference in survival between patients with a methylated MGMT Promoter (12 patients, 5 of whom died) and those with an unmethylated MGMT promoter (8 patients, 6 of whom died) was significant (P- 0.035 by the log-rank test), indicating that the MGMT methylation status has prognostic value.

Figure 1. Kaplan-Meier estimates of overall survival, according to MGMT promoter methylation status. 
Interestingly, we also found a subset of patients who received irinotecan and bevacizumab in the second line setting, where the unmethylated MGMT group of patients seemed to do better than the MGMT promoter methylated group. This finding has also been reported in other studies like the one done by Lamiss Mohamed A.E. et al. in Egypt who found that MGMT promoter unmethylated patients did better with irinotecan + bevacizumab-based treatment rather than standard temozolomide treatment [13]. However, larger studies are required to confirm the same.

\section{Conflicts of Interest}

The authors declare no conflicts of interest regarding the publication of this paper.

\section{References}

[1] Esteller, M., Garcia-Foncillas, J., Andion, E., et al. (2000) Inactivation of the DNA-Repair Gene MGMT and the Clinical Response of Gliomas to Alkylating Agents. The New England Journal of Medicine, 343, 1350-1354. https://doi.org/10.1056/NEJM200011093431901

[2] Hegi, M.E., Diserens, A.-C., Godard, S., et al. (2004) Clinical Trial Substantiates the Predictive Value of O-6-Methylguanine-DNA Methyltransferase Promoter Methylation in Glioblastom Apatients Treated with Temozolomide. Clinical Cancer Research, 10, 1871-1874. https://doi.org/10.1158/1078-0432.CCR-03-0384

[3] Ochs, K. and Kaina, B. (2000) Apoptosis Induced by DNA Damage O-6-Methylguanine Is Bcl-2 and Caspase-9/3 Regulated and Fas/Caspase- 8 Independent. Cancer Research, 60, 5815-5824.

[4] Liu, L., Markowitz, S. and Gerson, S.L. (1996) Mismatch Repair Mutations Override Alkyltransferase in Conferring Resistance to Temozolomide But Not to 1,3-bis(2chloroethyl) Nitrosourea. Cancer Research, 56, 5375-5379.

[5] Qian, X.C. and Brent, T.P. (1997) Methylation Hot Spots in the 5' Flanking Region Denote Silencing of the O-6-Methylguanine-DNA Methyltransferase Gene. Cancer Research, 57, 3672-3677.

[6] Watts, G.S., Pieper, R.O., Costello, J.F., Peng, Y.M., Dalton, W.S. and Futscher, B.W. (1997) Methylation of Discrete Regions of the O-6-Methylguanine DNA Methyltransferase (MGMT) CpG Island Is Associated with Heterochromatinization of the MGMT Transcription Start Site and Silencing of the Gene. Molecular and Cellular Biology, 17, 5612-5619. https://doi.org/10.1128/MCB.17.9.5612

[7] Esteller, M., Hamilton, S.R., Burger, P.C., Baylin, S.B. and Herman, J.G. (1999) Inactivation of the DNA Repair Gene O-6-Methylguanine-DNA Methyltransferase by Promoter Hypermethylation Is a Common Event in Primary Human Neoplasia. Cancer Research, 59, 793-797.

[8] Louis, D.N., Ohgaki, H., Wiestler, O.D. and Cavenee, W.K. (2007) WHO Classification of Tumours of the Central Nervous System. WHO Press (IARC), Lyon.

[9] Stupp, R., Mason, W.P., van den Bent, M.J., et al. (2005) Radiotherapy plus Concomitant and Adjuvant Temozolomide for Glioblastoma. The New England Journal of Medicine, 352, 987-996. https://doi.org/10.1056/NEJMoa043330

[10] Barker, F.G., Prados, M.D., Chang, S.M., et al. (1996) Radiation Response and Survival Time in Patients with Glioblastoma Multiforme. Journal of Neurosurgery, 84, 
442-448. https://doi.org/10.3171/jns.1996.84.3.0442

[11] Friedman, H.S., Pluda, J., Quinn, J.A., et al. (2000) Phase I Trial of Carmustine plus O6-Benzylguanine for Patients with Recurrent or Progressive Malignant Glioma. Journal of Clinical Oncology, 18, 3522-3528. https://doi.org/10.1200/JCO.2000.18.20.3522

[12] Quinn, J.A., Weingart, J., Brem, H., et al. (2003) Phase I Trial of Temozolomide plus O6-Benzylguanine in the Treatment of Patients with Recurrent or Progressive Cerebral Anaplastic Gliomas. Journal of Clinical Oncology, 22, 103.

[13] Lamiss Mohamed, A.E., Elkady, A.M., et al. (2018) Non-Methylated MGMT as Predictive Factor in Newly Diagnosed Glioblastoma Multiforme Treated with Bevacizumab Concurrent with Radiotherapy Followed by Adjuvant Bevacizumab plus Irinotecan versus Temozolomide Concurrent with Radiotherapy Followed by Adjuvant Temozolomide. Archives in Cancer Research, 6, 11.

https://doi.org/10.21767/2254-6081.100177 